\title{
Mechanics of wafer bonding: Effect of clamping
}

\author{
K. T. Turnera) \\ Massachusetts Institute of Technology, Cambridge, Massachusetts 02139 \\ M. D. Thouless \\ University of Michigan, Ann Arbor, Michigan 48109 \\ S. M. Spearing \\ Massachusetts Institute of Technology, Cambridge, Massachusetts 02139
}

(Received 20 August 2003; accepted 7 October 2003)

\begin{abstract}
A mechanics-based model is developed to examine the effects of clamping during wafer bonding processes. The model provides closed-form expressions that relate the initial geometry and elastic properties of the wafers to the final shape of the bonded pair and the strain energy release rate at the interface for two different clamping configurations. The results demonstrate that the curvature of bonded pairs may be controlled through the use of specific clamping arrangements during the bonding process. Furthermore, it is demonstrated that the strain energy release rate depends on the clamping configuration and that using applied loads usually leads to an undesirable increase in the strain energy release rate. The results are discussed in detail and implications for process development and bonding tool design are highlighted. (C) 2004 American Institute of Physics.
\end{abstract}

[DOI: $10.1063 / 1.1629776]$

\section{INTRODUCTION}

Wafer bonding has emerged as a key manufacturing process in a range of applications, including the commercial production of silicon-on-insulator (SOI) substrates, ${ }^{1}$ fabrication and packaging of microelectromechanical systems (MEMS), ${ }^{2,3}$ and efforts to develop three-dimensional integrated circuits. ${ }^{4,5}$ The basic wafer bonding process consists of joining wafers, which typically have thicknesses of $0.5-1$ $\mathrm{mm}$ and diameters from 100-300 mm, either directly or via an intermediate bonding layer. Among the wafer bonding processes commonly employed are direct, anodic, thermocompression, solder, glass frit, and polymer interlayer bonding. ${ }^{2}$ These processes are very different from one another and each of these technologies has specific attributes, such as processing temperature, mechanical strength, and process robustness, that may make them well suited for particular applications. While the bonding mechanisms in each of these processes are very different, the stresses and deformation that occur in the wafers during the bonding process are quite similar in all of them.

Fundamentally, wafer bonding requires the joining of two wafers, which most likely have slightly different shapes, to yield a single bonded pair. Semiconductor wafers tend to be very smooth, but may have hundreds of nanometers of flatness variation across millimeter-scale wavelengths (surface waviness and nanotopography), as well as wafer-scale shape variation of tens to hundreds of microns (bow and warp). To bond, the wafers must elastically deform to a common shape. This fact has been well recognized in the directbonding literature and there are several reports which look at the effect of surface waviness and wafer bow on direct bonding. ${ }^{6-8}$ While these works have examined flatness varia-

\footnotetext{
${ }^{a)}$ Electronic mail: kturner@mit.edu
}

tions, very little has been reported on the effect of loading and boundary conditions (i.e., applied clamping loads, mounting, chuck geometry, etc.) during the bonding process. One of the few reports on clamping is that by Feijoo et al. ${ }^{9}$ who proposed the idea of introducing curvature by deforming the wafers during bonding to increase the strain and hence the mobility of the device layer in SOI substrates. A curvature was introduced in the bonded pair by applying a load at the center during bonding and a model for the stress relaxation that occurs as one layer is thinned was presented. With the exception of that work, there has been no published work on interactions between the bonding tool and the bonded pair. This work seeks to address this by providing a model that permits the effect of basic clamping configurations to be understood. Specifically, the model that is developed provides a relationship between the initial shape and elastic properties of the wafers, and the final shape of the bonded pair and the strain energy release rate at the interface. Understanding the final shape of a bonded wafer pair is critical when the pair will undergo subsequent bonding steps or processing and handling. Furthermore, it is necessary to assess the effect that clamping may have on bonding failure, this is done by comparing the strain energy release rate to the bond toughness.

The two basic bonding configurations considered in this work are shown in Fig. 1. The first, which will be referred to as the free configuration, Fig. 1(a), is the traditional arrangement that is employed in direct and anodic bonding, in which surface forces (direct) or electrostatic forces (anodic) pull the wafers into contact. In this configuration, the wafers are initially contacted at a point, from which the bond spreads. No external loads are applied with the exception of the initial point contact. The other case considered, the clamped configuration, is shown in Fig. 1(b). This type of setup, which is 


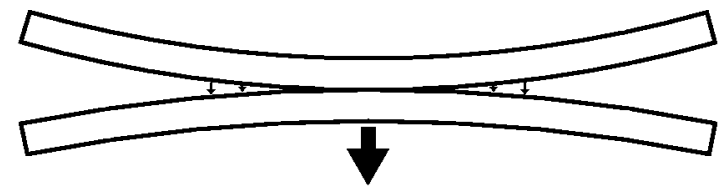

attractive forces pull wafers into contact

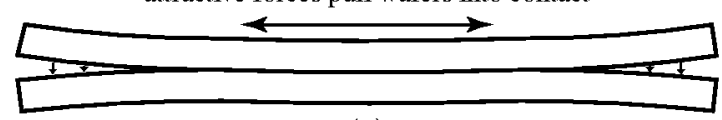

(a)

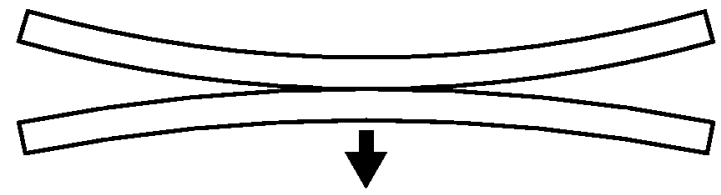

wafers pressed into contact by external load

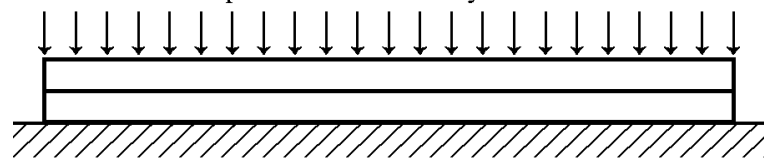

(b)

FIG. 1. Schematic of the two types of bonding processes considered in the current work. (a) Free configuration: Wafers are contacted at a point from which surface or electrostatic forces pull the wafers into contact. (b) Clamped configuration: Wafers are pressed into contact by the application of an external load.

common to many interlayer techniques, but may be used in direct and anodic bonding processes as well, presses the wafers into contact through the application of an external load. In the case considered here, the wafer pair is assumed to be supported by a chuck that defines the curvature during bonding. These two arrangements represent two bounding cases and serve as good models for understanding the basic effects of clamping in wafer bonding processes.

The structure of the article is as follows. First, the models for the two bonding cases are developed. The derivation is explained and expressions are developed for the final curvature of the bonded pair and the strain energy release rate at the interface. Next, the model is employed to understand how clamping may be used to improve the final shape of the bonded pair. Results from the free case and clamped case are compared and process implications highlighted. Then, the strain energy release rate results for the two cases are examined and compared. Finally, practical considerations are discussed and the results summarized.

\section{FREE CONFIGURATION}

The model system that is used to examine the free case, is illustrated schematically in Fig. 2. Two wafers, with geometry defined in terms of their curvatures, $\kappa_{1}, \kappa_{2}$, their thicknesses, $h_{1}, h_{2}$, and radius, $b$, are bonded by initially making contact at the center, from which the bond front propagates outward. A curvature that is concave up is defined as positive, as shown Fig. 2, a negative curvature indicates the wafer is concave down. The analysis for the free case is restricted to cases where $\kappa_{1} \geqslant \kappa_{2}$, as this is required in order to guarantee initial contact only at the center of the wafers. The wafers are taken to be elastic and isotropic, with Young's moduli, $E_{1}, E_{2}$, and Poisson's ratios, $\nu_{1}, \nu_{2}$. The

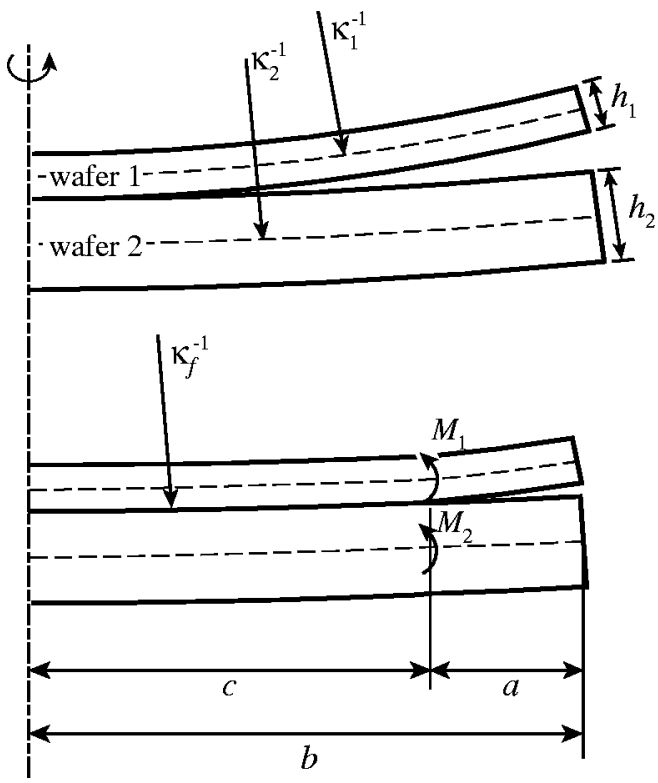

FIG. 2. Geometry and loading in the free case. Wafers with two different curvatures, which may be positive (concave up, as shown) or negative (concave down) are bonded and deform to a common curvature $\kappa_{f}$. The residual stress that results from the wafers being deformed from their initial curvatures to the final curvature may be treated as effective moments acting on each wafer.

crack length, which is measured from the edge of the wafer, is defined as $a$. As the wafers bond, each wafer is deformed from its initial curvature to a common final curvature, $\kappa_{f}$. As a result of this deformation, there is residual stress in the wafers following bonding. In the present case, the residual stress may be treated as an effective moment acting on each wafer. The moment acting on each wafer after bonding is

$$
M_{i}=\frac{1}{6} \bar{E}_{i} h_{i}^{3}\left(\kappa_{i}-\kappa_{f}\right) N_{i}(R),
$$

where the subscript $i$ denotes the wafer, either 1 or 2 , and $\bar{E}_{i}$ is the plane strain modulus, $\bar{E}_{i}=E_{i} /\left(1-\nu_{i}^{2}\right)$. The quantity, $N_{i}(R)$, is a nondimensional parameter that depends on the relative radius of the bonded area, $R=c / b$, and the Poisson's ratio,

$$
N_{i}(R)=\frac{\left(1+\nu_{i}\right)}{\left(1+\nu_{i}\right)+\left(1-\nu_{i}\right)(R)^{2}} .
$$

The expression given for the moment in each wafer by Eqs. (1) and (2) is derived from plate theory, as shown in Appendix A. Counterclockwise bending moments on right-hand side facing sections, as shown in Fig. 2, are defined as positive.

In the free case, no external loads are applied, thus at any radial position, equilibrium must be satisfied through a balance of stresses in the two wafers,

$$
M_{1}+M_{2}=0 \text {. }
$$

Using Eq. (1) with the equilibrium condition given by Eq. (3), the final curvature of the bonded stack can be determined

$$
\kappa_{f}=\frac{\Sigma \eta^{3} \lambda \kappa_{1}+\kappa_{2}}{\Sigma \eta^{3} \lambda+1} .
$$


Three nondimensional parameters have been defined to simplify the result, $\quad \Sigma=\bar{E}_{1} / \bar{E}_{2}, \quad \eta=h_{1} / h_{2}, \quad$ and $\lambda$ $=N_{1}(R) / N_{2}(R)$. The final curvature, in general, depends on the bonded radius because of the dependence on $\lambda$. However, when $\nu_{1}=\nu_{2}, \lambda=1$, and the final curvature is independent of the bond radius.

The residual stress that is present after bonding is stored elastic strain energy that may drive fracture processes, such as delamination, in the bonded pair. The strain energy release rate, $G$, at the interface may be expressed in terms of the moments and radial loads at the crack tip using the framework reported by Suo and Hutchinson. ${ }^{10}$ In the case under consideration here, where the only loads are the two moments $M_{1}$ and $M_{2}{ }^{11}$

$$
G=\frac{6}{\bar{E}_{1} h_{1}^{3}}\left(M_{1}^{2}+\Sigma \eta^{3} M_{2}^{2}\right) .
$$

Substitution into Eq. (5) of the moments, given by Eq. (1) with the final curvature defined by Eq. (4), yields the strain energy release rate

$$
G=\frac{1}{6} \bar{E}_{1} h_{1}^{3} \frac{\left(1+\Sigma \eta^{3}\right)}{\left(1+\Sigma \eta^{3} \lambda\right)^{2}}\left(\kappa_{2}-\kappa_{1}\right)^{2} N_{1}(R)^{2} .
$$

When the wafers have the same Poisson's ratio $\left(\nu_{1}=\nu_{2}\right.$ $=\nu)$, the expression for strain energy release rate reduces to

$$
\begin{aligned}
G= & \frac{1}{6} \frac{E_{1} h_{1}^{3}}{1+\Sigma \eta^{3}}\left(\kappa_{2}-\kappa_{1}\right)^{2} \frac{(1+\nu)}{(1-\nu)} \\
& \times \frac{1}{\left[(1+\nu)+(1-\nu) R^{2}\right]^{2}} .
\end{aligned}
$$

It should be noted that the expressions for final curvature and energy release rate of the free configuration derived in this analysis agree with those obtained in a previous analysis that was performed to investigate bond front propagation in direct-bonding processes when the wafers are initially bowed. ${ }^{8}$ In the previous analysis, bonding was examined, thus a quantity referred to as the strain energy accumulation rate was derived. This quantity is equivalent to the strain energy release rate that is derived in this work. In the earlier analysis, the strain energy was calculated using plate theory and the final curvature and strain energy release rate were determined through energy minimization and energy accounting, respectively. In the present analysis, effective moments on each layer are determined and the Suo and Hutchinson analysis for fracture in layered materials is employed, as this provides a more direct means of examining the clamped configuration. For the free case, there is no particular benefit to one analysis method over the other.

\section{CLAMPED CASE}

The second bonding scenario considered, the clamped case, is illustrated in Fig. 3. The two wafers are deformed to a common bonding curvature, $\kappa_{B}$, by the application of external loads. While being held at $\kappa_{B}$, the wafers are bonded. Following bonding, the clamping loads are removed and the wafer pair relaxes to its final curvature, $\kappa_{f}$. The analysis of

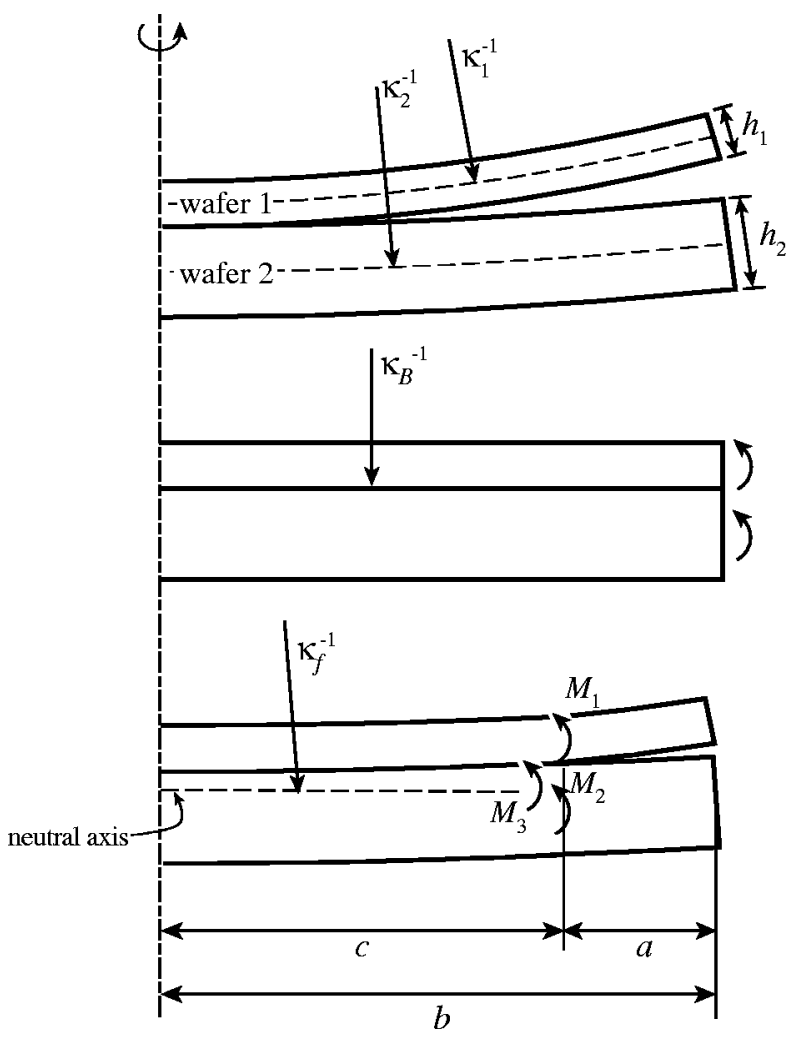

FIG. 3. Geometry and loading in the clamped case. Two wafers are deformed to a common curvature, $\kappa_{B}$, by external moments and then bonded. The external loads are then removed and the bonded stack relaxes to curvature $\kappa_{f}$.

this case is restricted to instances where, $\nu_{1}=\nu_{2}=\nu$, as it does not affect the results significantly, but does allow for considerable simplification in the derivation. Under this constraint, the moduli ratio reduces to, $\Sigma=E_{1} / E_{2}$.

The final curvature of the bonded pair once the loads are removed can be determined by considering the moments in the wafers. While the wafers are held at $\kappa_{B}$, there is a moment in each wafer,

$$
M_{i}=\frac{1}{6} \bar{E}_{i} h_{i}^{3}\left(\kappa_{i}-\kappa_{B}\right) N(R) .
$$

These internal moments in wafers 1 and 2 are initially balanced by the external loads. Once the wafers are bonded and the external loads are removed, the moments must be balanced by a moment in the bonded pair, $M_{3}$. Equilibrium requires

$$
M_{1}+M_{2}+M_{3}=0 .
$$

The moment acting on the bonded stack, $M_{3}$, causes the bonded pair to deform from $\kappa_{B}$ to $\kappa_{f}$. The bonded section may be treated as a composite plate, the moment-curvature relation for the bonded pair is

$$
\begin{aligned}
M_{3}= & \frac{1}{6} \bar{E}_{1} h_{1}^{3}\left(\kappa_{B}-\kappa_{f}\right) \\
& \times \frac{\left(\Sigma^{2} \eta^{4}+4 \Sigma \eta^{3}+6 \Sigma \eta^{2}+4 \Sigma \eta+1\right)}{(1+\Sigma \eta) \Sigma \eta^{3}} N(R) .
\end{aligned}
$$


The final curvature of the stack is determined by calculating $M_{3}$ from Eq. (9) by substituting for $M_{1}$ and $M_{2}$, which are given by Eq. (8),

$$
M_{3}=-\frac{1}{6} \bar{E}_{1} h_{1}^{3} \frac{1}{\Sigma \eta^{3}}\left[\Sigma \eta^{3} \kappa_{1}+\kappa_{2}-\left(\Sigma \eta^{3}+1\right) \kappa_{B}\right] N(R),
$$

then solving for the final curvature using Eqs. (10) and (11),

$$
\kappa_{f}=\frac{(\Sigma \eta+1)\left(\Sigma \eta^{3} \kappa_{1}+\kappa_{2}\right)+3 \Sigma \eta(\eta+1)^{2} \kappa_{B}}{\Sigma^{2} \eta^{4}+4 \Sigma \eta^{3}+6 \Sigma \eta^{2}+4 \Sigma \eta+1} .
$$

As for the free case, the strain energy release rate at the interface can be determined using the Suo and Hutchinson analysis for cracks in layered materials. In the current case, there are three moments applied at the crack tip, one on each layer and one that acts on the composite plate as shown in Fig. 3. The strain energy release rate for this case can be expressed as, ${ }^{11}$

$$
G=\frac{6}{\bar{E}_{1} h_{1}^{3}}\left(M_{1}^{2}+\Sigma \eta^{3} M_{2}^{2}-\frac{\Sigma}{12 I} M_{3}^{2}\right),
$$

where $I$ is the nondimensional moment of inertia given by

$$
I=\frac{1}{12} \frac{\Sigma^{2} \eta^{4}+4 \Sigma \eta^{3}+6 \Sigma \eta^{2}+4 \Sigma \eta+1}{\eta^{3}(\Sigma \eta+1)} .
$$

Substitution for $M_{1}$ and $M_{2}$, given by Eq. (8), and $M_{3}$, given by Eq. (11) into Eq. (13) yields the strain energy release rate at the interface for the clamped case,

$$
\begin{aligned}
G= & \frac{1}{6} \bar{E}_{1} h_{1}^{3} \frac{1}{\Sigma \eta^{3}}\left[\Sigma \eta^{3}\left(\kappa_{1}-\kappa_{B}\right)^{2}+\left(\kappa_{2}-\kappa_{B}\right)^{2}\right. \\
& \left.-\frac{(\Sigma \eta+1)\left(\Sigma \eta^{3} \kappa_{1}+\kappa_{2}-\left(\Sigma \eta^{3}+1\right) \kappa_{B}\right)^{2}}{\Sigma^{2} \eta^{4}+4 \Sigma \eta^{3}+6 \Sigma \eta^{2}+4 \Sigma \eta+1}\right] N(R)^{2} .
\end{aligned}
$$

This expression for strain energy release rate is valid assuming that after crack growth no contact occurs between the crack faces. Contact between the crack faces may occur when $\kappa_{1}<\kappa_{2}$.

\section{RESULTS AND DISCUSSION}

When examining the effect of wafer geometry and mounting on bonded wafer pairs there are two primary concerns: (1) The final shape of the bonded pair and (2) whether or not the bond will fail through delamination at the interface. The former is particularly important in devices that require the bonding of multiple wafers, since a large curvature in a bonded pair may lead to failure in subsequent bonding processes. Delamination is obviously always a concern in bonded structures and may be avoided by ensuring the interfacial strain energy release rate is less than the bond toughness. In this section, the results from the analysis above are used to examine both of these issues to provide practical guidance in process and equipment design.

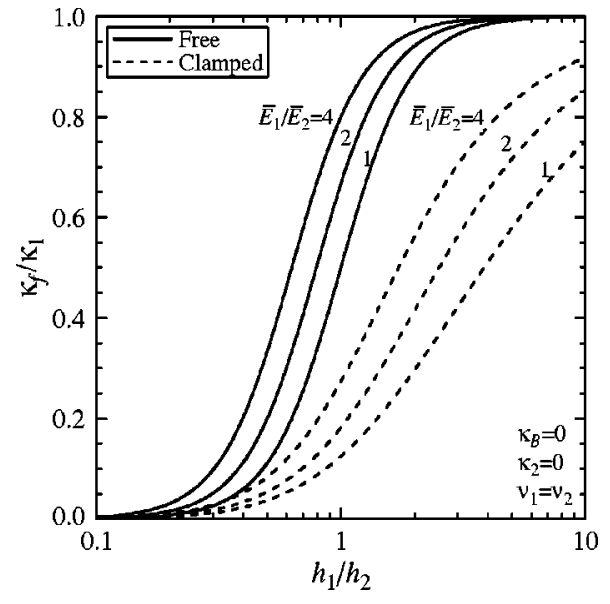

FIG. 4. Final curvature of a bonded wafer pair for the two different mounting cases. Plotted for various thickness and modulus ratios for the case where $\kappa_{2}=0$. For the clamped case, the bonding curvature is $\kappa_{B}=0$.

\section{A. Final shape}

The final shape of the bonded stack is described by the final curvature, $\kappa_{f}$, which is given by Eq. (4) for the free case and Eq. (12) for the clamped case. When the wafers have equal Poisson's ratios, the final curvature of the free case is only a function of the initial curvatures of the wafers, and the thickness and modulus ratios of the two wafers. For the clamped case, the final shape depends on one additional parameter, the bonding curvature. While the bonding curvature can be changed through different clamping configurations, typically a nominally flat chuck, corresponding to $\kappa_{B}$ $=0$, is employed.

The relative importance of the thickness and modulus ratio on the final curvature can be seen in Fig. 4. Figure 4 plots the final curvature for bonding an initially bowed wafer with curvature, $\kappa_{1}$ to a nominally flat wafer, $\kappa_{2}=0$, for the free and clamped case. From Fig. 4, it is clear that the relative thickness of the wafers is more important than the moduli ratio in determining the final curvature of the bonded pair. In the free case, when one layer is more than three times the thickness of the other, the final curvature is essentially determined by the initial curvature of the thick wafer. This strong dependence on thickness is due to the fact that the deformation of the wafers is bending dominated and thus has a cubic dependence on the thickness. When the wafers are clamped, then bonded, the thickness ratio also plays an important role in determining the final shape, but the effect is less pronounced because of the influence of the clamping curvature.

Perhaps more importantly, Fig. 4 demonstrates that clamping can make a significant difference in the final shape of the bonded pair. When the wafers have equal thickness and moduli ( $\eta=1, \Sigma=1)$ and the wafers are bonded in the free configuration, $\kappa_{f}=1 / 2 \kappa_{1}$. However, when the wafers are clamped flat and then bonded, $\kappa_{f}=1 / 8 \kappa_{1}$. This is a significant reduction in the curvature of the bonded pair solely due to way in which the wafers are mounted and demonstrates the potential for the use of clamping to control the shape of bonded pairs. 


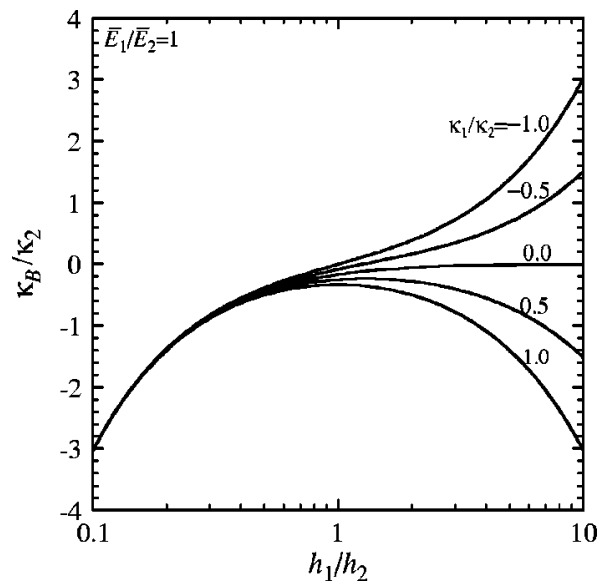

FIG. 5. The bonding curvature that will yield a flat bonded pair, $\kappa_{f}=0$, for various initial curvature as a function of thickness ratio.

The results in Fig. 4 are for the specific case of $\kappa_{B}=0$, which reduces the final curvature but, in general, does not result in a flat bonded pair. From Eq. (12), it is clear that if the initial geometry and material properties of the wafers are known, it is possible to select a bonding curvature that will yield a flat bonded pair. This idea is explored in Fig. 5, which plots the bonding curvature that is required to obtain a flat pair, $\kappa_{f}=0$, for various thickness and initial curvature ratios. The curvature ratio is defined as $\chi=\kappa_{1} / \kappa_{2}$, with $\left|\kappa_{1}\right|$ $\leqslant\left|\kappa_{2}\right|$ (the flatter of the two wafers is wafer 1). With this definition, the range of $\chi=-1.0$ to $\chi=1.0$, which corresponds to wafers that have equal but opposite curvature to wafers that have the same curvature, covers the full range of possibilities. As seen in Fig. 5, the bonding curvatures required to achieve a flat bonded pair are on the order of the initial curvatures of the wafers thus, in most cases, it is reasonable to achieve these through clamping. It is important to note though that as one wafer becomes much thinner than the other, the required bonding curvatures increase significantly, suggesting that it may be difficult to employ this method of curvature reduction in cases where one layer is very thin.

From the previous discussion, it is evident that clamping can be advantageous by reducing curvature in bonded pairs. There are situations however, in which poor clamping configurations may add curvature to a bonded pair. Figure 6 shows the final curvature when two initially flat wafers are bonded while deformed to curvature $\kappa_{B}$. When the wafers have equal thickness and modulus, the final curvature is $\kappa_{f}$ $=3 / 4 \kappa_{B}$. It is clear that a significant portion of the bonding curvature is retained after bonding. The bonded pair relaxes more as one of the wafers becomes thinner than the other. While this case may seem a bit obscure, it is actually an important scenario and demonstrates the importance of using a flat chuck when bonding initially flat wafers in a clamped configuration. This type of effect has been observed in practice, as reported in Ref. 12. Wafers, which were nominally flat, were bonded in a clamped configuration using two different chucks. In one case, a steel chuck was used and, in the other; a teflon chuck. Wafer pairs bonded using the teflon chuck had a significant curvature while those bonded on the steel chuck were nominally flat. The teflon chuck was not

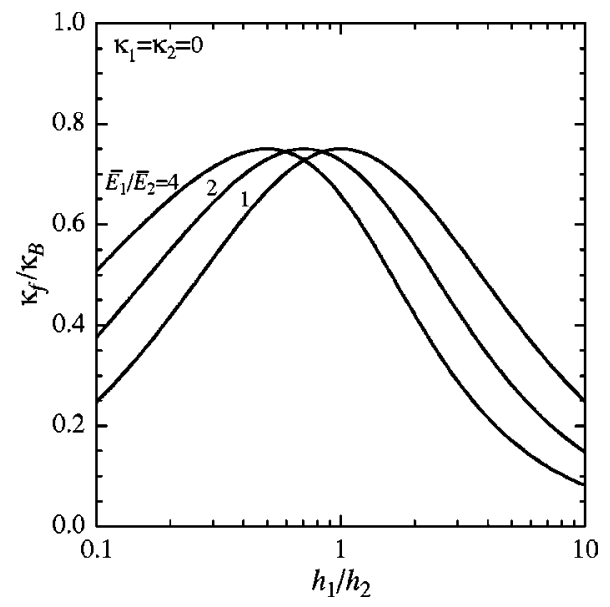

FIG. 6. The final curvature of pair bonded at curvature $\kappa_{B}$ when both wafers are initially flat $\left(\kappa_{1}=\kappa_{2}=0\right)$ for different modulus ratios as a function of thickness ratio.

initially as flat as the steel chuck and was also thought to be deforming more than the steel chuck under the applied loads, due to its low relative stiffness. As a result, the wafers bonded using the teflon chuck were being bonded in a curved state and retained significant shape after bonding, similar to the case plotted in Fig. 6. The model results along with this experimental example, demonstrate the need to use a sufficiently flat and stiff chuck when bonding flat wafers in a clamped configuration.

\section{B. Strain energy release rate}

As demonstrated above, the final curvature of a wafer bonded pair may be controlled through appropriate clamping and mounting during the bonding process. However, the clamping arrangement also influences the residual stresses in the bonded pair. If there is sufficient residual stress following bonding the wafers may delaminate. As such, when considering different clamping configurations, it is critical not only to consider the final shape, but the interfacial strain energy release rate as well.

The strain energy release rate for the free and clamped configuration are given by Eqs. (6) and (15), respectively. While the two have different dependencies on the moduli and thicknesses of the wafers, they both have the same dependence on crack length. The variation of the strain energy release rate with crack length is a function of the Poisson's ratio. The dependence on crack length is plotted for various values of Poisson's ratio in Fig. 7. The strain energy release rate increases with crack length suggesting that if a crack initiates, it will propagate unstably and the wafers will delaminate completely. It should be noted that the expressions for strain energy release rate given are not valid at very short crack lengths, $a \rightarrow 0$, or at short ligament lengths, $a$ $\rightarrow b$, when the characteristic dimension controlling the energy release rate ceases to be the plate thickness.

To assess the potential for delamination, the strain energy release rate must be compared to the interface toughness, $\Gamma$. Given that that the crack will propagate unstably if a crack initiates at the edge, a delamination criterion may be 


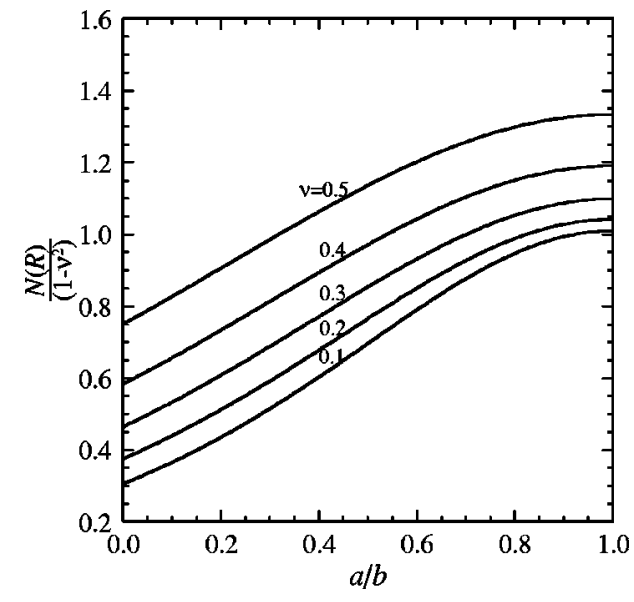

FIG. 7. Dependence of strain energy release rate on crack length for different values of Poisson's ratio. The free and clamped configurations have the same dependence on crack length, but have different magnitudes.

written by comparing the interface toughness to the strain energy release rate at $R=1$. When the wafers have the same elastic properties, the delamination criterion for the free case is

$$
\Gamma \leqslant \frac{1}{24} E h_{1}^{3} \frac{1+\nu}{1-\nu}\left[\frac{\left(\kappa_{2}-\kappa_{1}\right)^{2}}{1+\eta^{3}}\right],
$$

and for the clamped case (with $\kappa_{B}=0$ ) is

$$
\Gamma \leqslant \frac{1}{24} E h_{1}^{3} \frac{1+\nu}{1-\nu}\left[\frac{\eta^{3} \kappa_{1}^{2}+\kappa_{2}^{2}}{\eta^{3}}-\frac{\left(\eta^{3} \kappa_{1}+\kappa_{2}\right)^{2}}{\eta^{3}(1+\eta)^{3}}\right] .
$$

From Eqs. (16) and (17), it is clear that the strain energy release rate for the clamped and free case are markedly different. Figures 8 and 9 compare the strain energy release rate for the free and clamped (with $\kappa_{B}=0$ ) configuration. Figure 8 plots the normalized values of strain energy release rate, while Fig. 9 plots the ratio of the strain energy release rate for the free case to that of the clamped case. From Fig. 9, it is seen that the strain energy release rate in a pair bonded in the free configuration is always less than or equal to that of a

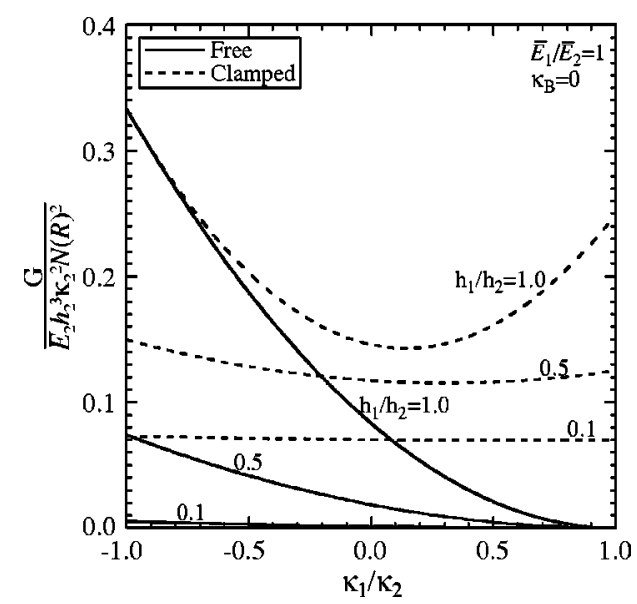

FIG. 8. Normalized strain energy release rate as a function of curvature ratio for the clamped and free case at several different thickness ratios. The wafers are taken to have the same elastic properties and the bonding curvature is fixed at $\kappa_{B}=0$.

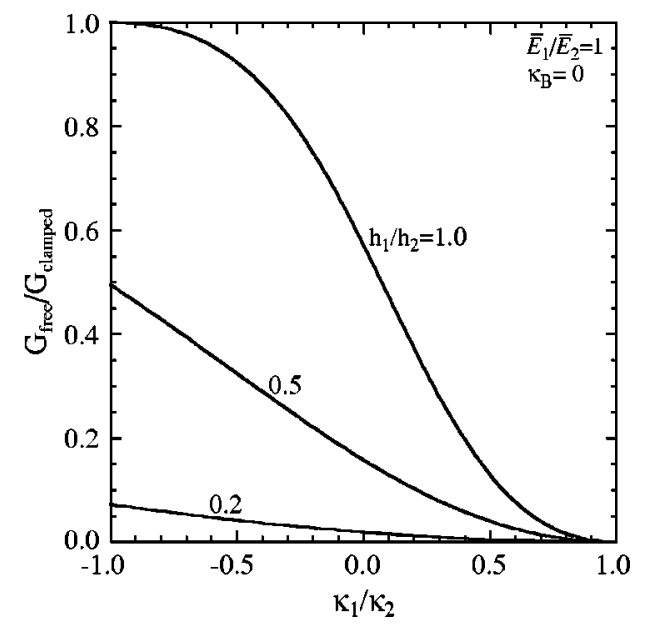

FIG. 9. Ratio between the strain energy release rate of the free and clamped cases plotted in Fig. 8.

pair bonded in a clamped configuration. In general, the only situation in which the strain energy release rate for the two cases are equal for a given pair of wafers is when the final curvature of the free case is equal to the bonding curvature in the clamped case. As seen in Fig. 9, the largest penalty is paid for clamping when the wafers have similar curvatures $\left(\kappa_{1} / \kappa_{2} \rightarrow 1\right)$. In the free case, the strain energy release rate is very low because the final curvature will be close to the initial curvature and the residual stresses in the wafers are small. However, in the clamped configuration, the bonding curvature may be far from the initial curvatures of the wafers, thus requiring more deformation of the wafers, resulting in a higher strain energy release rate.

These results suggest that care should be taken in selecting the clamping configuration for bonding processes. The fact that clamping the wafers during bonding tends to increase the strain energy release rates demonstrates that for processes, such as anodic and direct bonding, where clamping is not required, using applied clamping loads may result in an increase in bonding failure. There are processes however, such as thermocompression bonding, which require clamping pressure in order to form a bond, thus the use of applied loads is unavoidable. It is clear though that the way in which these loads are applied (i.e., selecting the bonding curvature) can be chosen to minimize the residual stresses and hence the strain energy release rate of the wafer pair.

A final important point to note is that the strain energy release rate that results due to clamping and flatness variations of the type described for a typical silicon wafer $(h$ $=0.5-1.0 \mathrm{~mm}, \kappa=0.01-0.1 \mathrm{~m}$, and $E=150 \mathrm{GPa}$ ) are on the order of 1 to $100 \mathrm{~mJ} / \mathrm{m}^{2}$. This is rather small, and well below the toughness of the majority of bonding techniques, thus in many cases clamping could be used to flatten bonded wafers as described in Sec. IV A without concern of introducing sufficient residual stress to cause delamination. Notably, the one technique where strain energy release rates of this magnitude may be significant is direct wafer bonding. In direct bonding, wafers are initially joined at room temperature and the bond toughness prior to annealing for a typical silicon-silicon pair is on the order of $10-100 \mathrm{~mJ} / \mathrm{m}^{2},{ }^{13}$ thus 
instances may occur where the use of clamping loads could lead to bonding failure.

\section{SUMMARY}

A mechanics analysis has been presented which describes the effect of two different clamping configurations during the bonding process. Given the initial geometry and elastic properties of the wafers as well as the bonding configuration, the final shape and strain energy release rate at the interface may be determined using the model presented. The results of the model demonstrate that the bonding curvature can influence the final shape of the bonded pair significantly. Using specific mounting configurations may permit bonded stacks to be engineered flat when the starting wafers are initially curved. The tooling required to achieve this is not standard at the present time, but the benefits of producing flat bonded pairs for subsequent bonding and processing may justify the effort required to develop it. The strain energy release rate results show that a pair bonded in the clamped configuration is usually more likely to delaminate than a pair bonded in the free configuration. The clamping process results in a larger amount of residual stress and hence a higher strain energy release rate. In processes where clamping pressure is required to form the bond, such as thermocompression and polymer interlayer techniques, the clamping curvature may be chosen to minimize the strain energy release rate. The magnitude of the strain energy release rate though, is well below the interface toughness achieved in most wafer bonding processes, with the exception of room-temperature direct bonding. Thus, clamping can often be employed to flatten wafers without a significant penalty. These results may be used to help guide the process design and equipment development in order to improve yield and performance in wafer bonding processes.

\section{ACKNOWLEDGMENTS}

Two of the authors (M. D. T. and S. M. S.) gratefully acknowledge N. A. Fleck for hosting them at Cambridge University during the period when this work was performed. This work was supported by the Cambridge MIT Institute [CMI-059/P-IR(FT)MEMS].

\section{APPENDIX}

The geometry considered is defined in Fig. 2. The curvature in the bonded section $(0 \leqslant r \leqslant c)$, section $\mathrm{A}$, is specified to be $\kappa_{f}$. Thus, the deflection from the initial curvature, $\kappa_{i}$, to the final curvature is

$$
w_{\mathrm{A}}=\frac{1}{2}\left(\kappa_{f}-\kappa_{i}\right) r^{2} \text {. }
$$

In the unbonded section $(c \leqslant r \leqslant b)$, section $\mathrm{B}$, there is no shear force, thus the governing equation for the plate is

$$
\frac{d}{d r}\left[\frac{1}{r} \frac{d}{d r}\left(r \frac{d w}{d r}\right)\right]=0
$$

Integrating the above equation and solving subject to the three boundary conditions,

$$
\begin{aligned}
& \left.w_{\mathrm{A}}\right|_{r=c}=\left.w_{\mathrm{B}}\right|_{r=c}, \\
& \left.\frac{d w_{\mathrm{A}}}{d r}\right|_{r=c}=\left.\frac{d w_{\mathrm{B}}}{d r}\right|_{r=c}, \\
& \left.M_{\mathrm{A}}\right|_{r=b}=0,
\end{aligned}
$$

the deflection in section $\mathrm{B}$ can be determined. Using the deflections, the moments in each section may be determined,

$$
M=-\frac{E h^{3}}{12\left(1-\nu^{2}\right)}\left(\frac{d^{2} w}{d r^{2}}+\frac{\nu}{r} \frac{d w}{d r}\right) .
$$

At $r=c$, equilibrium must be satisfied, thus the applied moment, $M$, is related to the moments in sections $\mathrm{A}$ and $\mathrm{B}$,

$$
M=-M_{\mathrm{B}}+M_{\mathrm{A}} \text {. }
$$

From this, the applied moment is found to be related to the curvature difference in the bonded section

$$
M=\frac{1}{6} \frac{E h^{3}}{(1-\nu)}\left(\kappa_{f}-\kappa_{i}\right) \frac{1}{(1+\nu)+(1-\nu)(c / b)^{2}} .
$$

One should note, that while the derivation above and throughout the article is concerned with the radial component of the moment, in the bonded section, the moment state is equi-biaxial.

${ }^{1}$ G. K. Celler and S. Cristoloveanu, J. Appl. Phys. 93, 4955 (2003).

${ }^{2}$ M. A. Schmidt, Proc. IEEE 86, 1575 (1998).

${ }^{3}$ A. R. Mirza and A. A. Ayon, Sensors 15, 24 (1998).

${ }^{4}$ K. Banerjee, S. Souri, P. Kapur, and K. Saraaswat, Proc. IEEE 89, 602 (1998).

${ }^{5}$ A. Fan, A. Rahman, and R. Reif, Electrochem. Solid-State Lett. 2, 534 (1999).

${ }^{6}$ Q.-Y. Tong and U. Gösele, J. Electrochem. Soc. 142, 3975 (1995).

${ }^{7}$ H. H. Yu and Z. Suo, J. Mech. Phys. Solids 46, 829 (1998).

${ }^{8}$ K. T. Turner and S. M. Spearing, J. Appl. Phys. 92, 7658 (2002).

${ }^{9}$ D. Feijoó, I. Ong, K. Mitani, W.-S. Yang, S. Yu, and U. M. Gösele, in Proceedings of the First International Symposium on Semiconductor Wafer Bonding: Science, Technology, and Applications (Electrochemical Society, New York, 1992), Vols. 92-97, pp. 230-238.

${ }^{10}$ Z. Suo and J. Hutchinson, Int. J. Fract. 43, 1 (1990).

${ }^{11}$ J. W. Hutchinson and Z. Suo, Adv. Appl. Mech. 29, 63 (1992).

${ }^{12}$ N. Miki, X. Zhang, R. Khana, A. A. Ayón, D. Ward, and S. M. Spearing, Sens. Actuators A 103, 194 (2003).

${ }^{13}$ Q.- Y. Tong and U. Gosele, Semiconductor Wafer Bonding: Science and Technology (Wiley, New York, 1999). 